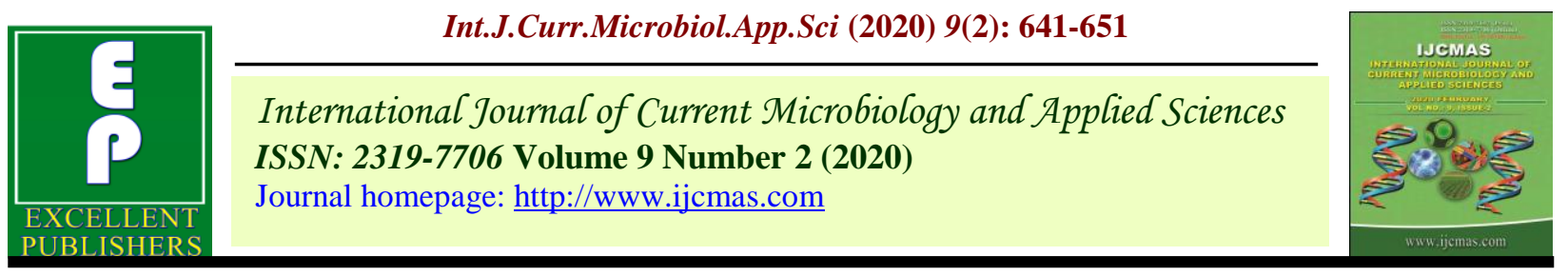

Original Research Article

https://doi.org/10.20546/ijcmas.2020.902.079

\title{
Nutrient Management Productivity and its Balance in Soybean (Glycine max (L) Merrill) Wheat (Triticum aestivum) Cropping in Vindhyan Plateau of MP, India
}

\author{
Praveen K. Jaga ${ }^{1^{*}}$ and G. S. Jamliya ${ }^{2}$ \\ ${ }^{1}$ Department of Soil Science and Agricultural Chemistry, ${ }^{2}$ Department of Agronomy, \\ JNKVV, College of Agriculture, GanjBasoda-464221(Vidisha), India \\ *Corresponding author
}

\begin{abstract}
A B S T R A C T
A field experiment was conducted during rainy (kharif) season of 2017-18 at farmers field at Sehore, (M.P), to study the effect of 8 fertilizer levels (75\% RDF, 75\% RDF + FYM $5 \mathrm{t}$ $\mathrm{ha}^{-1}, 100 \%$ RDF, $100 \%$ RDF + FYM $5 \mathrm{t} \mathrm{ha}^{-1}, 125 \%$ RDF, 125\% RDF + FYM $5 \mathrm{t} \mathrm{ha}^{-1}$, FYM $5 \mathrm{t} \mathrm{ha}^{-1}$, Absolute control) on Yield, nodulation, NPK content, uptake and its balance in two soybean varieties JS 95-60 and JS 97-52 were evaluated in factorial randomized block design with 3 replications. The grain yield was significantly higher with application of $125 \%$ RDF + FYM $5 \mathrm{t} \mathrm{ha}^{-1}\left(1820 \mathrm{~kg} \mathrm{ha}^{-1}\right)$, which was at par with $100 \%$ RDF + FYM $5 \mathrm{t}$ $\mathrm{ha}^{-1}\left(1801 \mathrm{~kg} \mathrm{ha}^{-1}\right)$. The variety JS 95-60 recorded significantly higher grain yield (2203 kg $\left.\mathrm{ha}^{-1}\right)$ as compared to variety JS 97-52 (886 kg ha $\left.{ }^{-1}\right)$. Number of root nodules per plant and dry weight of root nodules per plant are significantly higher with the application of $125 \%$ RDF + FYM $5 \mathrm{t} \mathrm{ha}^{-1}$ than other fertilizer treatments. Variety JS 97-52 was better in number and dry weight of root nodules per plant than JS 95-60. The NPK content and its uptake were significantly increased with to application of $125 \% \mathrm{RDF}+\mathrm{FYM} 5 \mathrm{t} \mathrm{ha}^{-1}$ followed by $100 \%$ RDF + FYM $5 \mathrm{t} \mathrm{ha}^{-1}$. Variety JS 95-60 was also noticed significantly increased in respect of $\mathrm{N}, \mathrm{P}$ and $\mathrm{K}$ content in straw, seed and their uptake. Application of different treatment combinations of fertilizer levels and varieties improved the available $\mathrm{N}$ in the soil. Phosphorus and potassium showed marginal gain in sum experimental plots.
\end{abstract}

\section{Introduction}

Soybean is one of the important legume crops of India which not only helps in maintaining soil fertility but it is also a rich source of protein and fats. Oil and protein rich soybean has now been recognized all over the world as a potential supplementary source of edible oil and nutrition. The oil of soybean contains $85 \%$ unsaturated fatty acid and is cholesterol free. Soybean seeds contain $43.2 \%$ protein, $19.5 \%$ fat, $20.9 \%$ carbohydrate and a good amount of other nutrients like calcium, phosphorus, iron and vitamins (Gopalan et al., 1971). Soybean has 3\% lecithin which is helpful for brain development. The appropriate combination of mineral fertilizers with organic manure can be feasible and visible to sustain agriculture as commercial and profitable ensuring high yield of crop 
without deterioration in quantity and quality of the produce and soil health. FYM (Farm yard Manure) is by far, the most popular and available for use as an organic source of plant nutrient with the farmers. The use of FYM is the tool to improve the physical, chemical and biological properties of the soil. Farmyard manure being the source of all essential elements, improves soil organic matter and humus part of soil. FYM also plays an important role inhabitation beneficial bacteria thus making the nutrients available to crop. Therefore the present study was undertaken to see the effect of applied inorganic fertilizer with FYM and without FYM on Yield, nodulation, NPK content, uptake and its balance in soybean varieties.

\section{Materials and Methods}

The field experiment was conducted during the rainy (kharif) season 2017-18 at farmers field of Sehore, Madhya Pradesh $\left(23^{0} 12^{\prime} \mathrm{N}\right.$ latitude, $77^{0} 05^{\prime} \mathrm{E}$ longitude and at $498.77 \mathrm{~m}$ above mean sea level). The experimental site is characterized by sub-tropical zone with extreme temperature during summer $\left(45.60^{\circ} \mathrm{c}\right)$ and very low temperature (as low as $5^{\circ} \mathrm{c}$ ). The average rainfall varies from 1000 to $1200 \mathrm{~mm}$ most of which is received during JuneSeptember. The soil was medium black clay loam having $\mathrm{pH}$ (7.3), electrical conductivity (EC) $\left(0.12 \mathrm{dS} \mathrm{m}^{-1}\right)$, organic carbon (0.58), medium available $\mathrm{N}\left(245.25 \mathrm{~kg} \mathrm{ha}^{-1}\right)$, medium available $\mathrm{P}\left(17.80 \mathrm{~kg} \mathrm{ha}^{-1}\right)$, and high available $\mathrm{K}$ (425.24 $\left.\mathrm{kg} \mathrm{ha}^{-1}\right)$. The field trail consisting of 8 levels of fertilizer (75\% RDF, $75 \%$ RDF + FYM $5 \mathrm{t} \mathrm{ha}^{-1}, 100 \%$ RDF, $100 \% \mathrm{RDF}+$ FYM $5 \mathrm{t} \mathrm{ha}^{-1}, 125 \%$ RDF, $125 \%$ RDF +FYM $5 \mathrm{t} \mathrm{ha}^{-1}$, FYM $5 \mathrm{t} \mathrm{ha}^{-1}$, Absolute control) and 2 levels of soybean variety (JS 95-60 and JS 9752) was a factorial experiment laid out in randomized block design with 3 replications. Fertilizer doses were calculated on the basis of recommended dose of nutrients $20 \mathrm{~N}+60$ $\mathrm{P}_{2} \mathrm{O}_{5}+20 \mathrm{~K}_{2} \mathrm{O}+20 \mathrm{~S}, \mathrm{~kg} \mathrm{ha}{ }^{-1}$ through urea, single super phosphate and muriate of potash, respectively. The quantity of fertilizer was calculated for respective treatment separately and was drilled as per treatment at the time of sowing. Decomposed farm yard manure (FYM)@ 5 tones ha $^{-1}$ were applied before sowing and well incorporated in the soil. The sowing was carried out on $5^{\text {th }}$ July 2010 maintaining row spacing of $45 \mathrm{~cm}$ with recommended seed rate of JS 95-60 and JS 97-52 was $75 \mathrm{~kg} \mathrm{ha}^{-1}$ and $65 \mathrm{~kg} \mathrm{ha}^{-1}$, respectively was applied. Other agronomic management practices were followed as per the standard recommendation. The crop harvested at maturity and observation on Yield, straw yield and nodulation were recorded as per standard procedure. The soil samples collected after harvest of soybean crop were analysed for available N (Subbiah and Asija 1956), $\mathrm{P}$ (Olsen et al., 1954) and K (Hanway and Heidel 1952). The grain and straw samples were digested with di acid mixture of $\mathrm{HNO}_{3}$ and $\mathrm{HClO}_{4}$ in 9:1ratio. Phosphorous was determined by vanadomolybdate yellow colour method (Jackson, 1973), K by flame photometer. Nitrogen in plants was determined by modified micro Kjeldahl method. The nutrient uptake was calculated by multiplying the nutrient content values with the yield data. The data were statistically analysed using standard procedures of ANOVA at 5\% level of significance.

\section{Results and Discussion}

\section{Number and weight of root nodules}

Fertilizer levels increased the number and dry weight of root nodules per plant (Table 1). The maximum number and weight of root nodules per plant were recorded with the application of $125 \% \mathrm{RDF}+\mathrm{FYM} 5 \mathrm{t} \mathrm{ha}^{-1}$ which might be due to better root development and profuse nodulation on account of increase in the rhizobial activity in 
the rhizosphere under fertilizer levels especially due to increased $\mathrm{P}$ availability, which resulted in the formation of active and more number of root nodules. The results are in close agreement with the findings of Lone et al., (2009) and Mohod et al., (2010). The variety JS 97-52 was significantly superior in number of root nodules and dry weight of root nodules per plant than variety JS 95-60.

\section{Grain and straw yields}

Fertilizer levels were significantly influenced the grain yield of soybean (Table 1). Application of $125 \% \mathrm{RDF}+\mathrm{FYM} 5 \mathrm{t} \mathrm{ha}^{-1}$ was significantly higher in grain yield and it was at par with $100 \%$ RDF + FYM $5 \mathrm{t} \mathrm{ha}^{-1}$. This might be due to balanced use of fertilizers in soil which increased their availability in soil. The increment in supply of essential elements through organic and inorganic sources, their availability, mobilization and influx into the plant tissues increased and thus improved growth and yield components and finally the seed yield of soybean. The results corroborated the findings of Singh Guriqbal (2009) and Shinde et al., (2009). The variety JS 95-60 produced highest grain yield $\left(2203 \mathrm{~kg} \mathrm{ha}^{-1}\right)$ than variety JS 97-52 (886 kg ha-1). It may be due to higher seeds per pod and better seed index. These favourable phenomenon in this variety resulted higher seed yield. Similar results were also observed by Thakur and Vyas (2005), and Khan et al., (2005) were also reported variation in yield attributed with different varieties. Maximum straw yield was obtained in $125 \%$ RDF + FYM $5 \mathrm{t} \mathrm{ha}^{-1}$ (3127 $\left.\mathrm{kg} \mathrm{ha}{ }^{-1}\right)$. It may be due to better growth attributes. Tiwari et al., (1997) observed that the maximum straw yield of 5.94 tonnes was obtained with the application of 100 per cent $\mathrm{NPK}+5 \mathrm{t} \mathrm{ha}^{-1}$ FYM. The variety JS 97-52 produced significantly higher straw yield $\left(3576 \mathrm{~kg} \mathrm{ha}^{-1}\right.$ ) compared to the variety JS 9560. The highest straw yield may be due to the plant height and higher number of branches and dry weight per plant. This favourable morphological phenomenon in this variety resulted highest straw yield.

\section{Nitrogen content $(\%)$}

The $\mathrm{N}$-content in straw and seed was influenced by different fertilizer levels (Table 2). Application of $125 \% \mathrm{RDF}+\mathrm{FYM} 5 \mathrm{tha}^{-1}$ was recorded significantly higher $\mathrm{N}$ - content in straw $(0.89 \%)$ and in seed $(6.51 \%)$ and it was at par with $100 \%$ RDF + FYM $5 \mathrm{t} \mathrm{ha}^{-1}$. It may be due to balanced and complete nutrition of plant through judicious combination of organics and in-organics. The beneficial effect of FYM must be due to its role in adequate nutrient supply, enhanced mobilization of nutrients, activation of beneficial soil microbes, biological activities (N-fixation) and improved physical condition of soil which provides the plant a good nutrient content to grow and develop. Effect of varieties on $\mathrm{N}$ - content in straw was not significant but $\mathrm{N}$-content in seed was significant. The variety JS 95-60 was significantly superior in $\mathrm{N}$ - content in seed than variety JS 97-52. Total uptake of nitrogen by soybean (straw + seed) showed (Table 3) similar trend as it was seen and recorded in case of straw and seed contents. The increase in uptake of nitrogen could be the results of enhanced physiological processes within the plant system which resulted in the increased absorption of nitrogen by soybean plant and thereby, translocation of nitrogen. Supplementation of FYM with in-organic fertilizer improves the crop growth and thereby uptake of nitrogen. These results are in close conformity of the results observed by Chaturvedi and Chandel (2005) and Lone et al., (2009).

\section{Phosphorus content (\%)}

Application of $125 \% \mathrm{RDF}+\mathrm{FYM} 5 \mathrm{t} \mathrm{ha}^{-1}$ recorded the highest $\mathrm{P}$-content in straw $(0.133 \%)$ and in seed $(0.87 \%)$ which were 
significantly superior than the other treatments (Table 2). This might be due to higher availability of plant nutrients with fertilization and continuous supply of nutrients through FYM, which resulted in increased nutrient content in the plant tissues and greater biomass production at higher fertilizer levels with FYM. It also may be synergistic effect between $\mathrm{N}$ and P. Similar findings in soybean crop was reported by Lone et al., (2009). The variety JS 95-60 recorded significantly higher P-content in straw and seed as compared to variety JS 9752. The total uptake was also improved significantly with different levels of fertilizer application and varieties (Table 3). The variety JS 95-60 recorded significantly higher total (straw + seed) P- uptake than JS 97-52. Application of $125 \%$ RDF + FYM $5 \mathrm{t} \mathrm{ha}^{-1}$ recorded the highest $\left(20.58 \mathrm{~kg} \mathrm{ha}^{-1}\right)$ total Puptake (straw +seed) which was significantly superior than other treatments but was at par with $100 \% \mathrm{RDF}+\mathrm{FYM} 5 \mathrm{tha}^{-1}$. The increase in uptake of phosphorus could be the results of enhanced physiological process within the plant system which resulted in the increased absorption of phosphorus by soybean plant and thereby, translocation of phosphorus which might have resulted in good yield of soybean. These results are in close conformity of the results observed by Chaturvedi and Chandel (2005) and Lone et al., (2009).

Table.1 Effect of fertilizer levels and varieties on number and dry weight of root nodules, grain yield $\left(\mathrm{kg} \mathrm{ha}^{-1}\right)$ and straw yield $\left(\mathrm{kg} \mathrm{ha}^{-1}\right)$

\begin{tabular}{|c|c|c|c|c|}
\hline Treatments & $\begin{array}{l}\text { Number of } \\
\text { root nodules } \\
\text { per plant }\end{array}$ & $\begin{array}{l}\text { Dry weight of } \\
\text { root nodules } \\
\text { per plant (g) }\end{array}$ & $\begin{array}{c}\text { Grain yield } \\
\left.\text { (kg ha-1 }^{-1}\right)\end{array}$ & $\begin{array}{c}\text { Straw yield } \\
\left(\mathrm{kg} \mathrm{ha}^{-1}\right)\end{array}$ \\
\hline \multicolumn{5}{|l|}{ Fertilizer levels (F) } \\
\hline $75 \%$ RDF & 44.94 & 111.38 & 1599 & 2955 \\
\hline $75 \%$ RDF + FYM 5 t ha-1 & 49.61 & 124.85 & 1657 & 2980 \\
\hline $100 \%$ RDF & 46.61 & 117.83 & 1710 & 3044 \\
\hline $100 \%$ RDF + FYM 5 t ha ${ }^{-1}$ & 51.44 & 130.86 & 1801 & 3081 \\
\hline $125 \%$ RDF & 50.17 & 126.98 & 1716 & 3069 \\
\hline $125 \%$ RDF + FYM $5 \mathrm{t} \mathrm{ha}^{-1}$ & 56.50 & 144.01 & 1820 & 3127 \\
\hline FYM 5 t ha ${ }^{-1}$ & 47.61 & 118.95 & 1054 & 2929 \\
\hline Control & 40.94 & 100.78 & 997 & 2875 \\
\hline SEm \pm & 0.63 & 1.61 & 33.67 & 63.30 \\
\hline $\mathrm{CD}(\mathrm{P}=\mathbf{0 . 0 5})$ & 1.81 & 4.64 & 96.97 & NS \\
\hline \multicolumn{5}{|l|}{ Varieties (V) } \\
\hline JS 95-60 & 47.03 & 118.75 & 2203 & 2439 \\
\hline JS 97-52 & 49.93 & 125.16 & 886 & 3576 \\
\hline SEm \pm & 0.31 & 0.80 & 16.84 & 31.65 \\
\hline $\mathrm{CD}(\mathrm{P}=\mathbf{0 . 0 5})$ & 0.90 & 2.32 & 48.49 & 91.58 \\
\hline
\end{tabular}


Table.2 Effect of fertilizer levels and varieties on NPK-content (\%) in straw and seed

\begin{tabular}{|c|c|c|c|c|c|c|}
\hline Treatments & $\begin{array}{l}\text { N-content } \\
(\%) \text { in } \\
\text { straw }\end{array}$ & $\begin{array}{l}\mathrm{N} \text {-content } \\
(\%) \text { in seed }\end{array}$ & $\begin{array}{c}\text { P-content } \\
(\%) \text { in } \\
\text { straw }\end{array}$ & $\begin{array}{l}\text { P-content } \\
(\%) \text { in seed }\end{array}$ & $\begin{array}{c}\text { K-content } \\
(\%) \text { in } \\
\text { straw }\end{array}$ & $\begin{array}{l}\text { K- } \\
\text { content } \\
(\%) \text { in } \\
\text { seed }\end{array}$ \\
\hline \multicolumn{7}{|l|}{ Fertilizer levels $(\mathrm{F})$} \\
\hline $75 \% \mathrm{RDF}$ & 0.80 & 6.35 & 0.100 & 0.74 & 1.17 & 2.44 \\
\hline $75 \%$ RDF + FYM $5 \mathrm{t} \mathrm{ha}^{-1}$ & 0.84 & 6.40 & 0.106 & 0.79 & 1.27 & 2.52 \\
\hline $100 \% \mathrm{RDF}$ & 0.82 & 6.40 & 0.105 & 0.76 & 1.25 & 2.50 \\
\hline $100 \%$ RDF + FYM $5 \mathrm{t} \mathrm{ha}^{-1}$ & 0.86 & 6.46 & 0.122 & 0.84 & 1.37 & 2.61 \\
\hline $125 \% \mathrm{RDF}$ & 0.85 & 6.44 & 0.115 & 0.81 & 1.35 & 2.59 \\
\hline $125 \%$ RDF + FYM $5 \mathrm{t} \mathrm{ha}^{-1}$ & 0.89 & 6.51 & 0.133 & 0.87 & 1.47 & 2.69 \\
\hline FYM $5 \mathrm{t} \mathrm{ha}^{-1}$ & 0.78 & 6.30 & 0.095 & 0.70 & 1.13 & 2.40 \\
\hline Control & 0.76 & 6.05 & 0.085 & 0.65 & 1.07 & 2.30 \\
\hline SEm \pm & 0.01 & 0.028 & 0.004 & 0.007 & 0.01 & 0.022 \\
\hline $\mathrm{CD}(\mathrm{P}=0.05)$ & 0.02 & 0.079 & 0.011 & 0.02 & 0.03 & 0.063 \\
\hline \multicolumn{7}{|l|}{ Varieties (V) } \\
\hline JS 95-60 & 0.83 & 6.44 & 0.114 & 0.86 & 1.38 & 2.66 \\
\hline JS 97-52 & 0.82 & 6.28 & 0.101 & 0.68 & 1.13 & 2.35 \\
\hline SEm \pm & 0.0033 & 0.014 & 0.0019 & 0.004 & 0.005 & 0.011 \\
\hline $\mathrm{CD}(\mathrm{P}=0.05)$ & NS & 0.040 & 0.0054 & 0.011 & 0.014 & 0.031 \\
\hline
\end{tabular}


Table.3 Effect of fertilizer levels and varieties on NPK-uptake $\left(\mathrm{kg} \mathrm{ha}^{-1}\right)$ in straw, seed and total (straw + seed)

\begin{tabular}{|c|c|c|c|c|c|c|c|c|c|}
\hline Treatments & $\begin{array}{l}\text { N-uptake } \\
\left(\mathrm{kg} \mathrm{ha}^{-1}\right) \text { in } \\
\text { straw }\end{array}$ & $\begin{array}{l}\text { N-uptake } \\
\left(\mathrm{kg} \mathrm{ha}^{-1}\right) \text { in } \\
\text { seed }\end{array}$ & $\begin{array}{l}\text { Total N- } \\
\text { uptake }(\mathrm{kg} \\
\left.\text { ha }^{-1}\right) \text { in } \\
\text { straw + } \\
\text { seed }\end{array}$ & $\begin{array}{l}\text { P-uptake } \\
\left(\mathrm{kg} \mathrm{ha}^{-1}\right) \text { in } \\
\text { straw }\end{array}$ & $\begin{array}{l}\text { P-uptake } \\
\left(\mathrm{kg} \mathrm{ha}^{-1}\right) \text { in } \\
\text { straw }\end{array}$ & $\begin{array}{l}\text { Total P- } \\
\text { uptake }(\mathrm{kg} \\
\left.\text { ha }^{-1}\right) \text { in } \\
\text { straw + } \\
\text { seed }\end{array}$ & $\begin{array}{l}\text { K-uptake } \\
\left(\mathrm{kg} \mathrm{ha}^{-1}\right) \text { in } \\
\text { straw }\end{array}$ & $\begin{array}{l}\text { K-uptake } \\
\left(\mathrm{kg} \mathrm{ha}^{-1}\right) \text { in } \\
\text { straw }\end{array}$ & $\begin{array}{l}\text { Total K- } \\
\text { uptake }(\mathrm{kg} \\
\left.\text { ha }^{-1}\right) \text { in } \\
\text { straw + } \\
\text { seed }\end{array}$ \\
\hline \multicolumn{10}{|l|}{ Fertilizer levels $(\mathrm{F})$} \\
\hline $75 \% \mathrm{RDF}$ & 23.44 & 102.17 & 125.66 & 2.94 & 12.41 & 15.35 & 33.57 & 40.05 & 73.62 \\
\hline $75 \%$ RDF + FYM $5 \mathrm{t} \mathrm{ha}^{-1}$ & 24.62 & 106.65 & 131.27 & 3.14 & 13.70 & 16.84 & 37.50 & 42.89 & 80.39 \\
\hline $100 \% \mathrm{RDF}$ & 24.96 & 110.16 & 135.12 & 3.17 & 13.82 & 17.00 & 36.63 & 44.02 & 80.85 \\
\hline $100 \% \mathrm{RDF}+\mathrm{FYM} 5 \mathrm{t} \mathrm{ha}^{-1}$ & 26.57 & 116.90 & 143.47 & 3.70 & 15.80 & 19.50 & 42.72 & 48.30 & 91.03 \\
\hline $125 \% \mathrm{RDF}$ & 25.95 & 111.11 & 137.06 & 3.50 & 14.51 & 18.00 & 41.17 & 45.66 & 86.82 \\
\hline $125 \%$ RDF + FYM $5 \mathrm{t} \mathrm{ha}^{-1}$ & 27.73 & 119.07 & 146.80 & 4.08 & 16.50 & 20.58 & 42.73 & 50.25 & 92.98 \\
\hline FYM $5 \mathrm{t} \mathrm{ha}^{-1}$ & 22.85 & 66.53 & 89.38 & 2.75 & 7.73 & 10.49 & 32.42 & 25.90 & 58.32 \\
\hline Control & 21.67 & 60.57 & 82.23 & 2.41 & 6.86 & 9.27 & 32.44 & 23.32 & 55.76 \\
\hline $\mathrm{SEm} \pm$ & 0.56 & 2.17 & 2.49 & 0.12 & 0.26 & 0.31 & 0.92 & 0.92 & 1.10 \\
\hline $\mathrm{CD}(\mathrm{P}=0.05)$ & 1.61 & 6.25 & 7.18 & 0.35 & 0.75 & 0.88 & 2.65 & 2.66 & 3.18 \\
\hline \multicolumn{10}{|l|}{ Varieties (V) } \\
\hline JS 95-60 & 20.17 & 142.48 & 162.67 & 2.80 & 19.17 & 21.97 & 34.18 & 59.10 & 93.29 \\
\hline JS 97-52 & 29.27 & 55.81 & 85.08 & 3.63 & 6.16 & 9.78 & 40.61 & 21.00 & 61.60 \\
\hline $\mathrm{SEm} \pm$ & 0.28 & 1.08 & 1.24 & 0.06 & 0.13 & 0.15 & 0.46 & 0.46 & 0.55 \\
\hline $\mathrm{CD}(\mathrm{P}=0.05)$ & 0.80 & 3.13 & 3.59 & 0.18 & 0.38 & 0.44 & 1.32 & 1.33 & 1.59 \\
\hline
\end{tabular}

Table.4 Balance of nitrogen in soil as influenced by fertilizer levels and varieties 
Int.J.Curr.Microbiol.App.Sci (2020) 9(2): 641-651

\begin{tabular}{|c|c|c|c|c|c|c|c|}
\hline Treatments & $\begin{array}{l}\text { N-added } \\
\text { through } \\
\text { fertilizer } \\
\left(\mathrm{kg} \mathrm{ha}^{-1}\right)\end{array}$ & Total N & $\begin{array}{c}\text { Crop } \\
\text { removal } \mathrm{N} \\
\left(\mathrm{kg} \mathrm{ha}^{-1}\right)\end{array}$ & $\begin{array}{c}\text { Theoretical } \mathrm{N} \\
\text { balance } \\
\left(\mathrm{kg} \mathrm{ha}^{-1}\right)\end{array}$ & $\begin{array}{l}\text { Actual } \mathrm{N} \text { after } \\
\text { harvesting }(\mathrm{kg} \\
\left.\text { ha }^{-1}\right)\end{array}$ & $\begin{array}{c}\text { Loss } \\
\text { / gain of } \\
\text { available } \mathrm{N} \\
\left(\mathrm{kg} \mathrm{ha}^{-1}\right)\end{array}$ & $\begin{array}{c}\text { Loss/gain of } \mathrm{N} \\
\text { over initial } \\
\left(\mathrm{kg} \mathrm{ha}^{-1}\right)\end{array}$ \\
\hline JS 95-60 + 75\% RDF & 15 & 260.25 & 171.21 & 89.04 & 250.37 & 161.33 & 5.12 \\
\hline JS 95-60 + $75 \%$ RDF + FYM & 40 & 285.25 & 173.98 & 111.27 & 253.91 & 142.64 & 8.66 \\
\hline JS $95-60+100 \%$ RDF & 20 & 265.25 & 183.46 & 81.79 & 251.25 & 169.46 & 6.00 \\
\hline JS $95-60+100 \%$ RDF + FYM & 45 & 290.25 & 188.77 & 101.48 & 255.75 & 154.27 & 10.50 \\
\hline JS $95-60+125 \%$ RDF & 25 & 270.25 & 177.83 & 92.42 & 253.60 & 161.18 & 8.35 \\
\hline JS $95-60+125 \%$ RDF + FYM & 50 & 295.25 & 186.70 & 108.55 & 257.27 & 148.72 & 12.02 \\
\hline JS 95-60 + FYM & 25 & 270.25 & 114.31 & 155.94 & 252.30 & 98.36 & 7.05 \\
\hline JS 95-60 + Absolute Control & - & 245.25 & 105.06 & 140.19 & 247.03 & 104.18 & 1.78 \\
\hline JS $97-52+75 \%$ RDF & 15 & 260.25 & 80.11 & 180.14 & 256.02 & 75.88 & 10.77 \\
\hline JS $97-52+75 \%$ RDF + FYM & 40 & 285.25 & 88.55 & 196.7 & 260.25 & 63.55 & 15.00 \\
\hline JS $97-52+100 \%$ RDF & 20 & 265.25 & 86.78 & 178.47 & 258.75 & 80.28 & 13.50 \\
\hline JS $97-52+100 \%$ RDF + FYM & 45 & 290.25 & 98.17 & 192.08 & 261.50 & 69.42 & 16.25 \\
\hline JS $97-52+125 \%$ RDF & 25 & 270.25 & 96.29 & 173.96 & 259.30 & 85.34 & 14.05 \\
\hline JS $97-52+125 \%$ RDF + FYM & 50 & 295.25 & 106.91 & 188.34 & 265.45 & 77.11 & 20.20 \\
\hline JS 97-52 + FYM & 25 & 270.25 & 64.44 & 205.81 & 260.00 & 54.19 & 14.75 \\
\hline JS 97-52 + Absolute Control & - & 245.25 & 59.41 & 185.84 & 247.36 & 61.52 & 2.11 \\
\hline
\end{tabular}

* Initial-N $245.25 \mathrm{~kg} \mathrm{ha}^{-1}$ 


\section{Int.J.Curr.Microbiol.App.Sci (2020) 9(2): 641-651}

Table.5 Balance of phosphorus in soil as influenced by fertilizer levels and varieties

\begin{tabular}{|c|c|c|c|c|c|c|c|}
\hline Treatments & $\begin{array}{l}\text { P-added } \\
\text { through } \\
\text { fertilizer } \\
\left(\mathrm{kg} \mathrm{ha}^{-1}\right)\end{array}$ & Total P & $\begin{array}{c}\text { Crop } \\
\text { removal P } \\
\left(\mathrm{kg} \mathrm{ha}^{-1}\right)\end{array}$ & $\begin{array}{c}\text { Theoretical } \mathrm{P} \\
\text { balance } \\
\left(\mathrm{kg} \mathrm{ha}^{-1}\right)\end{array}$ & $\begin{array}{c}\text { Actual P after } \\
\text { harvesting }(\mathrm{kg} \\
\left.\mathrm{ha}^{-1}\right)\end{array}$ & $\begin{array}{l}\text { Loss / gain } \\
\text { of available } \\
\mathrm{P}\left(\mathrm{kg} \mathrm{ha}^{-1}\right)\end{array}$ & $\begin{array}{c}\text { Loss/gain of P } \\
\text { over initial } \\
\left(\mathrm{kg} \mathrm{ha}^{-1}\right)\end{array}$ \\
\hline JS $95-60+75 \%$ RDF & 45 & 62.8 & 21.88 & 40.92 & 13.97 & -26.95 & -3.83 \\
\hline $\mathrm{JS} 95-60+75 \% \mathrm{RDF}+\mathrm{FYM}$ & 55 & 72.8 & 23.55 & 49.25 & 14.23 & -35.02 & -3.57 \\
\hline JS $95-60+100 \%$ RDF & 60 & 77.8 & 24.35 & 53.45 & 15.23 & -38.22 & -2.57 \\
\hline JS $95-60+100 \%$ RDF + FYM & 70 & 87.8 & 27.09 & 60.71 & 15.51 & -45.20 & -2.29 \\
\hline JS $95-60+125 \%$ RDF & 75 & 92.8 & 24.56 & 68.24 & 16.00 & -52.24 & -1.80 \\
\hline JS 95-60 + $125 \%$ RDF + FYM & 85 & 102.8 & 27.73 & 75.07 & 16.87 & -58.20 & -0.93 \\
\hline JS 95-60 + FYM & 10 & 27.8 & 14.05 & 13.75 & 16.61 & -0.14 & -4.19 \\
\hline JS 95-60 + Absolute Control & - & 17.8 & 12.57 & 5.23 & 8.20 & 2.97 & -9.60 \\
\hline JS $97-52+75 \%$ RDF & 45 & 62.8 & 8.82 & 53.98 & 14.51 & -39.47 & -3.29 \\
\hline JS $97-52+75 \%$ RDF + FYM & 55 & 72.8 & 10.13 & 62.67 & 14.87 & -47.80 & -2.93 \\
\hline $\mathrm{JS} 97-52+100 \% \mathrm{RDF}$ & 60 & 77.8 & 9.65 & 68.15 & 15.94 & -52.21 & -1.86 \\
\hline JS $97-52+100 \%$ RDF + FYM & 70 & 87.8 & 11.99 & 75.89 & 16.18 & -59.71 & -1.62 \\
\hline $\mathrm{JS} 97-52+125 \% \mathrm{RDF}$ & 75 & 92.8 & 11.45 & 81.35 & 16.46 & -64.89 & -1.34 \\
\hline JS $97-52+125 \%$ RDF + FYM & 85 & 102.8 & 13.43 & 89.37 & 17.23 & -72.14 & -0.57 \\
\hline JS 97-52 + FYM & 10 & 27.8 & 6.92 & 20.88 & 14.44 & -6.44 & -3.36 \\
\hline JS 97-52 + Absolute Control & - & 17.8 & 5.97 & 11.83 & 9.50 & -2.33 & -8.30 \\
\hline
\end{tabular}

* Initial-P $17.8 \mathrm{~kg} \mathrm{ha}^{-1}$ 
Table.6 Balance of potassium in soil as influenced by fertilizer levels and varieties

\begin{tabular}{|c|c|c|c|c|c|c|}
\hline Treatments & $\begin{array}{c}\text { K-added } \\
\text { through } \\
\text { fertilizer }(\mathrm{kg} \\
\left.\mathrm{ha}^{-1}\right)\end{array}$ & Total K & $\begin{array}{c}\text { Crop } \\
\text { removal K } \\
\left(\mathrm{kg} \mathrm{ha}^{-1}\right)\end{array}$ & $\begin{array}{c}\text { Theoretical K } \\
\text { balance }(\mathrm{kg} \\
\left.\text { ha }^{-1}\right)\end{array}$ & $\begin{array}{l}\text { Actual } \mathrm{K} \text { after } \\
\text { harvesting }(\mathrm{kg} \\
\left.\mathrm{ha}^{-1}\right)\end{array}$ & $\begin{array}{l}\text { Loss/gain of } \mathrm{K} \\
\text { over initial (kg } \\
\left.\mathrm{ha}^{-1}\right)\end{array}$ \\
\hline JS $95-60+75 \%$ RDF & 15 & 440.24 & 91.72 & 348.52 & 390.40 & -34.84 \\
\hline JS 95-60 + $75 \%$ RDF + FYM & 40 & 465.24 & 96.87 & 368.37 & 399.57 & -25.67 \\
\hline JS $95-60+100 \%$ RDF & 20 & 445.24 & 101.26 & 343.98 & 392.10 & -33.14 \\
\hline JS 95-60 + $100 \%$ RDF + FYM & 45 & 470.24 & 109.63 & 360.61 & 402.22 & -23.02 \\
\hline JS $95-60+125 \%$ RDF & 25 & 450.24 & 103.37 & 346.87 & 393.75 & -31.49 \\
\hline JS 95-60 + $125 \%$ RDF + FYM & 50 & 475.24 & 110.31 & 364.93 & 405.56 & -19.68 \\
\hline JS 95-60 + FYM & 25 & 450.24 & 67.06 & 383.18 & 393.98 & -31.26 \\
\hline JS 95-60 + Absolute Control & - & 425.24 & 66.09 & 359.15 & 382.36 & -42.88 \\
\hline JS $97-52+75 \%$ RDF & 15 & 440.24 & 55.52 & 384.72 & 394.17 & -31.07 \\
\hline JS 97-52 + $75 \%$ RDF + FYM & 40 & 465.24 & 63.90 & 401.34 & 403.87 & -21.37 \\
\hline JS $97-52+100 \%$ RDF & 20 & 445.24 & 60.05 & 385.19 & 396.87 & -28.37 \\
\hline JS 97-52 + $100 \%$ RDF + FYM & 45 & 470.24 & 72.42 & 397.82 & 408.34 & -16.90 \\
\hline JS $97-52+125 \%$ RDF & 25 & 450.24 & 70.27 & 379.97 & 398.60 & -26.64 \\
\hline JS $97-52$ + $125 \%$ RDF + FYM & 50 & 475.24 & 75.65 & 399.59 & 410.58 & -14.66 \\
\hline JS 97-52 + FYM & 25 & 450.24 & 49.58 & 400.66 & 397.24 & -28.00 \\
\hline JS 97-52 + Absolute Control & - & 425.24 & 45.43 & 379.81 & 385.60 & -39.64 \\
\hline
\end{tabular}

* Initial-K $425.24 \mathrm{~kg} \mathrm{ha}^{-1}$ 


\section{Potassium content (\%)}

Application of $125 \% \mathrm{RDF}+\mathrm{FYM} 5 \mathrm{t} \mathrm{ha}^{-1}$ recorded significantly higher $\mathrm{K}$-content in straw $(1.47 \%)$ and in seed $(2.69 \%)$ than other treatments (Table 2). The trend of such results could be ascribed due to the initial higher availability of potassium in the experimental soil, indicating the luxury consumption of potassium. The variety JS 95-60 recorded significantly higher K-content in straw and in seed as compared to variety JS 97-52. The total uptake was also improved significantly with different levels of fertilizers application and varieties (Table 3). The variety JS 95-60 recorded significantly higher total (straw + seed) K- uptake than variety JS 97-52. Application of $125 \% \mathrm{RDF}+\mathrm{FYM} 5 \mathrm{t} \mathrm{ha}^{-1}$ recorded the highest $\left(92.98 \mathrm{~kg} \mathrm{ha}^{-1}\right)$ total $\mathrm{K}$ uptake (straw +seed) which was significantly superior to other treatments but was at par with $100 \%$ RDF + FYM 5 t ha $^{-1}$. It might be due to combined application of organics and in-organics which ultimately enhanced $\mathrm{K}$ absorption by plants. These results are in close conformity of the results observed by Chaturvedi and Chandel (2005) and Singh et al., (2007).

\section{Nitrogen, phosphorus and potassium balance in soil}

The data on soil available $\mathrm{N}$ indicated in (Table 4) showed a positive balance of nitrogen in soil in almost all the plots as compared to initial level of available nitrogen $\left(245.25 \mathrm{~kg} \mathrm{ha}^{-1}\right)$. The actual nitrogen left in soil was ranged from 2.11 to $20.20 \mathrm{~kg} \mathrm{ha}^{-1}$. It was maximum $\left(20.20 \mathrm{~kg} \quad \mathrm{ha}^{-1}\right)$ with combination of variety JS 97-52 + 125\% RDF + FYM $5 \mathrm{t} \mathrm{ha}^{-1}$ and minimum was in variety JS 97-52+ no fertilizer. The increase in actual $\mathrm{N}$ left in the soil may be due to increased nodulation and thereby symbiotic $\mathrm{N}$ fixation, and application of organic matter (FYM) along with in-organic fertilizer. Singh et al.,
(1998) reported that incorporation of FYM and inoculation of seed left a net balance of $40 \mathrm{~kg} \mathrm{~N} \mathrm{ha}{ }^{-1}$ for succeeding crop.

The net phosphorus balance in soil showed a negative trend in (Table 5) all most all the plots as influenced by different levels of fertilizer application and varieties. The reduction in net available phosphorus was lesser under variety JS 97-52 + 125\% RDF + FYM $5 \mathrm{t} \mathrm{ha}^{-1}$. The depletion of $\mathrm{P}$ might be due to more removable of $\mathrm{P}$ from furrow layers, poor availability and fixation of $\mathrm{P}$. The results are in close agreement with the findings of Lal et al., (1997).

The data on the net potassium balance in soil showed a negative trends in (Table 6) almost all the treatments, which may be due to more removable of $\mathrm{K}$ by soybean due to more complimentary uptake with $\mathrm{N}$ and $\mathrm{P}$ and less supplementation losses were observed when $\mathrm{K}$ was applied with $125 \% \mathrm{RDF}+\mathrm{FYM} 5 \mathrm{t}$ ha 1 . These results are in close agreement with the findings of Lal et al., (1997).

\section{References}

Chaturvedi, S. and Chandel, A.S. (2005). Influence of organic and inorganic fertilizer on soil fertility and productivity of soybean [Glycine max]. Indian Journal of Agronomy: 50(4): 311-313.

Gopalan, C., Sastri, R.B.V. and Balasubramanian, S.C. 1971. Nutritive value of Indian Foods. ICMR. 16(2): 62-63.

Hanway, J. and Heidal, H.S. (1952) Soil testing laboratory procedure. IOWA Agri. 57 (1): 31.

Jackson, M. L. (1973) Soil Chemical analysis, Prentice Hall Inc. England Cliffs, New Jersy.

Khan, Hakim, Bashir Mohammad and Amanullah (2005). Performance of full 
season soybean varieties at Mansehra. Sarhad J. Agri. 21 (3) 327-329

Lone, Bilal Ahmed, Hasan,Badrul, Ansar,S. and Singh , Amarjeet (2009) Effect of seed rate, row spacing and fertility levels on growth and nutrient uptake of soybean[Glycine max (L) Merrill] under temperature conditions. Journal of Agricultural and biological Science 4(3) :7-10.

Mohod, N.B., Nemade Seema , Ghadage Preeti (2010) Effect of integrated nutrient management on growth and yield parameters of soybean . Green Farming 1(3) : 270-271.

Olsen, S.R., Cole, C.V., Watanabe, F.S. and Dean, L.A. (1954) Estimation of available- $\mathrm{P}$ in soil by extraction with $\mathrm{NaHCO}_{3}$. United States Department of Agriculture Circular, 939:1-9.

Shinde, P.S., Sankpal, V.Y., Jawale, S.M., Shaikh, A.A., Dalavi, N.D. and Jadhav, M.B. (2009) Effect of integrated nutrient management on yield attributes and quality of soybean. Journal of Maharashtra Agricultural Universities 34(1): 107-108.
Singh Guriqbal (2009) Effect of FYM, Macro and micro-nutrients on the growth and grain yield of soybean. Journal of Plant Science Research 25 (2):155-158.

Singh, Muneshwar, Tripathi, A.K., Kundu, S. , Ramana, S. , Takkar, P.N. and Singh, M. (1998) Effect of seed inoculation and FYM on biological $\mathrm{N}$ fixation in soybean and nitrogen balance under soybean wheat system on vertisols. Indian Soc. Soil Sci. 46 (4): 604-609.

Subbaih, B.V. and Asija, G.L. (1956) A rapid method for the estimation of available nitrogen in soil. Curent Science. 25: 259-260.

Thakur, B.S. and Vyas, M.D. (2005) Relative performance of soybean (Glycine max (L) Merrill) varieties under varying plant population and row spacing. M.Sc. (Ag) Thesis, J.N.K.V.V., Jabalpur.

Tiwari, Alok, Sharma, S.K. Shrivastava, S.P. and Tambhare, B.R. (1997) Study of plant physiological growth parameters and yield of soybean (Glycine max (L) Merrill) under the influence of manure and fertilizer. Adv. Pl. Sci. 10 (1): 149152.

\section{How to cite this article:}

Praveen K. Jaga and Jamliya, G. S. 2020. Nutrient Management Productivity and its Balance in Soybean (Glycine max (L) Merrill) Wheat (Triticum aestivum) Cropping in Vindhyan Plateau of MP, India. Int.J.Curr.Microbiol.App.Sci. 9(02): 641-651.

doi: https://doi.org/10.20546/ijcmas.2020.902.079 